\title{
Morbimortalidade por Câncer de Próstata em Homens Adultos em um Estado do Nordeste do Brasil: Caracterização Epidemiológica e Análise de Tendência Temporal
}

\section{Morbimortality From Prostate Cancer in Adult Men in a State of Northeast Brazil: Epidemiological Characterization and Temporal Trend Analysis}

\section{RESUMO}

Objetivo: Traçar o perfil epidemiológico e analisar a tendência temporal de homens adultos com diagnóstico e/ou óbito por câncer de próstata no Estado de Alagoas, Brasil. Metodologia: Estudo ecológico, realizado a partir dos registros de morbimortalidade para câncer de próstata, em homens com idade entre 20 a 59 anos no Estado de Alagoas. Foram empregados os programas BioEstat 5.3 para avaliação das variáveis epidemiológicas e estimar a variação percentual anual da incidência e da mortalidade e seus respectivos intervalos de confiança. Resultados: Foi observado que 209 homens foram atendidos em virtude do câncer de próstata em Alagoas. Os casos ocorreram, predominantemente, em indivíduos de 55 a 59 anos, negros, com baixa escolaridade, que fizeram uso de álcool e/ou tabaco em algum momento da vida e que não apresentavam histórico familiar de câncer. Foi verificada a ocorrência 54 óbitos (25,8\%). Conclusão: O estudo viabilizou o conhecimento do perfil epidemiológico de homens adultos com diagnóstico e/ou óbito por câncer de próstata no Estado de Alagoas e das tendências temporais da incidência da mortalidade por câncer de próstata no período estudado.

\section{DESCRITORES}

Morbimortalidade. Câncer de Próstata. Saúde do Homem. Brasil.

\begin{abstract}
Objective: To trace an epidemiological profile and analysis of temporal trends in adult men who were diagnosed and/or died of prostate cancer in the state of Alagoas, Brazil. Methodology: Ecological study carried out from the records of morbidity and mortality for prostate cancer in men aged between 20 and 59 years in the state of Alagoas. The BioEstat 5.3 programs were used to assess epidemiological variables and estimate the annual percentage change in incidence and mortality and their respective confidence intervals. Results: It was observed that 209 men were treated for prostate cancer in Alagoas. The cases occurred predominantly in individuals aged 55 to 59 years, black, with low education, who used alcohol and/or tobacco at some point in their lives and who had no family history of cancer. 54 deaths $(25.8 \%)$ were found. Conclusion: The study enabled the knowledge of the epidemiological profile of adult men who were diagnosed and/or died of prostate cancer in the state of Alagoas and of the temporal trends in the incidence of prostate cancer mortality during the period studied.
\end{abstract}

\section{DESCRIPTORS}

Morbidity and Mortality. Prostate Cancer. Men's Health. Brazil.

\footnotetext{
${ }^{1}$ Acadêmico de Medicina da Universidade Estadual de Ciências da Saúde de Alagoas (UNCISAL), Mestrando em Ensino na Saúde pelo Programa de Mestrado em Ensino na Saúde da UNCISAL. Biólogo, especialista em Saúde Coletiva, Maceió, Alagoas, Brasil.

2 Professora Mestre do Núcleo de Ciências Humanas, Sociais e Políticas Públicas da Universidade Estadual de Ciências da Saúde de Alagoas (UNCISAL), Doutoranda em Saúde Pública pelo Programa de Pós-Graduação em Saúde Pública da USP, Maceió, Alagoas, Brasil.
} 
câncer de próstata (CaP) é considerado um relevante problema de saúde pública, especialmente entre os países de média e baixa renda, como é o caso do Brasil ${ }^{1}$. Esse status devese ao impacto social, à morbimortalidade e aos altos custos atrelados ao seu tratamento nos sistemas públicos e privados de saúde ${ }^{2,3}$.

Estimativas do Instituto Nacional do Câncer colocam essa patologia na qualidade da segunda neoplasia mais frequente entre os homens no país, ficando atrás apenas do câncer de pele e apontam para uma expectativa de cerca de 65.000 novos casos por ano no país ${ }^{4}$.

Tal contexto revela-se preocupante, principalmente, quando analisado sob o aspecto da evolução da doença, dos seus impactos econômicos e das perdas indiretas consequentes da incapacidade produtiva do acometido pela patologia 4 .

Apesar do CaP estar comumente relacionado a idades mais avançadas, estudos tem relatado, de forma cada vez mais frequente, a presença em homens a partir de 45 anos e até mesmo antes dessa faixa etária ${ }^{3-6}$. Considerando que $20 \%$ dos casos de CaP são diagnosticados em estágios avançados e uma média de $25 \%$ dos pacientes evoluem para óbito ${ }^{7}$, torna-se importante identificar as características e os fatores envolvidos no adoecimento desta população ${ }^{6}$, para que sejam executadas ações mais efetivas relacionadas ao diagnóstico precoce e ao enfretamento da doença, assim como o planejamento de ações que promovam melhoria das condições de saúde dos indivíduos ${ }^{8}$.

Partindo do pressuposto de que a incidência de CaP no Brasil tem se tornado mais frequente e que, em Alagoas, esse tipo de neoplasia urológica vem ocupando o primeiro lugar entre as causas de óbitos por câncer entre os indivíduos do sexo masculino ${ }^{5}$, faz-se necessária a investigação sobre a ocorrência deste agravo, bem como de seus fatores condicionantes, a fim de que se possa organizar ações e serviços de saúde capazes de gerar medidas de intervenção no âmbito da saúde do homem.

Assim, visando contribuir para o conhecimento da situação de saúde das populações, este estudo teve por objetivo traçar o perfil epidemiológico e realizar a análise de tendência temporal dos indivíduos com diagnóstico e/ou óbito por $\mathrm{CaP}$, na população de homens adultos do Estado de Alagoas, assistidos pelo Sistema Único de Saúde (SUS), de modo a identificar os fatores epidemiológicos relacionados com a patologia no território alagoano.

\section{METODOLOGIA}

Trata-se de um estudo ecológico de séries temporais, com caráter retrospectivo, que considera como unidade de análise o Estado de Alagoas, Brasil.

A população do estudo foi composta por todos os registros de morbimortalidade (diagnósticos e óbitos) por neoplasia urológica do tipo câncer de próstata (CID 10: C61), no período de $1^{\circ}$ de janeiro de 2010 a 31 de dezembro de 2015, em homens adultos, com idade entre 20 a 59 anos, notificados ao Registro Hospitalar de Câncer do Instituto Nacional do Câncer (RHC/INCA) e que receberam atendimento em alguma unidade 
hospitalar de Alagoas credenciada ao SUS. Os dados sobre mortalidade foram obtidos por meio do Sistema de Informações sobre Mortalidade (SIM/SUS), por intermédio da plataforma TABWIN/DATASUS. A coleta de dados foi realizada entre os meses dezembro de 2017 e abril de 2018. Optou-se pelo ano de 2015 como ponto de corte desta pesquisa pelo fato de, quando da etapa definida em cronograma para a realização da coleta de dados, esse ter sido o último ano em que as notificações do RHC/INCA tinham sido totalmente processadas.

A análise da morbidade por câncer de próstata no Estado de Alagoas, entre homens adultos, considerou como variáveis de investigação: faixa etária $(<40,40$ a 49, 50 a 59), cor da pele (branca, indígena, parda, preta, sem informação), escolaridade (nenhuma, até 9 anos de estudo, 9 anos ou mais, sem informação), estado conjugal (casado, solteiro, viúvo, sem informação), histórico de consumo de bebida (sim, nunca, ex-consumidor, sem informação) e tabaco (sim, nunca, ex-consumidor, sem informação), histórico de câncer na família (sim, não, sem informação).

A caracterização dos óbitos por câncer de próstata, por sua vez, foi realizada considerando as seguintes variáveis, em virtude da limitação de informações da fonte de dados: faixa etária $(<40,40$ a 44,45 a 49, 50 a 54, 55 a 59), cor da pele (branca, parda, preta, sem informação), escolaridade (nenhuma, menos de 8 anos, 8 anos ou mais, ignorado), estado civil (casado, solteiro, viúvo, outro, sem informação) e região de saúde de residência ( $1^{\mathrm{a}}$ a $10^{\mathrm{a}}$ região de saúde). Todas as informações foram organizadas considerando o ano de registro da informação na fonte de dados.

Os dados sociodemográficos foram compilados no programa Microsoft Office Excel 2010, sendo posteriormente transportados para o programa BioEstat, versão 5.3, onde foram analisados por meio da geração de indicadores descritivos e inferenciais das variáveis.

Por se tratar de estudo realizado com o uso de dados oriundos de fontes secundárias, não foi necessária a aprovação pelo Comitê de Ética em Pesquisa.

\section{RESULTADOS}

Foi observado entre os atendimentos hospitalares ocorridos no Estado de Alagoas, no período de 2010 a 2015, que 317 homens, com idade entre 20 e 59 anos, obtiveram diagnóstico de neoplasia maligna. Destes, verificou-se que 209 (65,9\%) foram diagnosticados como casos novos de câncer de próstata.

Na Tabela 1 é possível observar o perfil sociodemográfico dos casos novos de câncer de próstata em Alagoas, no período estudado. Verificou-se que entre os casos novos de câncer de próstata em homens de 20 a 59 anos, as maiores proporções foram encontradas na faixa etária de 50 a 59 anos (91,0\%), em indivíduos da raça/cor parda (78,9\%), com até nove anos de escolaridade $(44,5 \%)$, casados $(55,5 \%)$, com relato de ingestão de bebida alcoólica em algum momento da vida (39,8\%), consumo de tabaco em algum momento da vida $(43,3 \%)$ e sem histórico familiar de câncer $(45,0 \%)$.

Diante dos registros no sistema 
Tabela 1. Características sociodemográficas dos casos novos de câncer de próstata, em homens de 20 a 59 anos, no período de 2010 a 2015, Alagoas, Brasil

\begin{tabular}{|c|c|c|}
\hline Variáveis & $\mathrm{n}$ & $\mathrm{N}^{\circ}$ de casos novos de câncer de próstata (\%) \\
\hline TOTAL & 209 & 100,0 \\
\hline \multicolumn{3}{|l|}{ Faixa Etária } \\
\hline$<40$ & 04 & 1,9 \\
\hline 40 a 49 & 15 & 7,1 \\
\hline 50 a 59 anos & 190 & 91,0 \\
\hline \multicolumn{3}{|l|}{ Raça/Cor } \\
\hline Branca & 25 & 12,0 \\
\hline Indígena & 01 & 0,5 \\
\hline Parda & 165 & 78,9 \\
\hline Preta & 08 & 3,8 \\
\hline Sem Informação & 10 & 4,8 \\
\hline \multicolumn{3}{|l|}{ Escolaridade } \\
\hline Nenhuma & 27 & 12,9 \\
\hline Até 9 anos & 93 & 44,5 \\
\hline 9 anos ou mais & 38 & 18,2 \\
\hline Sem Informação & 51 & 24,4 \\
\hline \multicolumn{3}{|l|}{ Estado Civil } \\
\hline Casado & 115 & 55,0 \\
\hline Solteiro & 78 & 37,3 \\
\hline Viúvo & 06 & 2,9 \\
\hline Sem Informação & 10 & 4,8 \\
\hline \multicolumn{3}{|l|}{ Consumo de bebida } \\
\hline Sim & 29 & 13,9 \\
\hline Nunca & 64 & 30,8 \\
\hline Ex-consumidor & 54 & 25,9 \\
\hline Sem Informação & 61 & 29,4 \\
\hline \multicolumn{3}{|l|}{ Consumo de tabaco } \\
\hline Sim & 22 & 11,1 \\
\hline Nunca & 65 & 32,7 \\
\hline Ex-consumidor & 64 & 32,2 \\
\hline Sem Informação & 48 & 24,0 \\
\hline \multicolumn{3}{|c|}{ Histórico familiar de câncer } \\
\hline Sim & 48 & 23,0 \\
\hline Não & 94 & 45,0 \\
\hline Sem Informação & 67 & 32,0 \\
\hline
\end{tabular}

Fonte: Registro Hospitalar de Câncer do Instituto Nacional do Câncer (RHC/INCA).

Tabela 2. Tendência da taxa de incidência de casos de câncer de próstata, em homens de 20 a 59 anos, no período de 2010 a 2015, Alagoas, Brasil

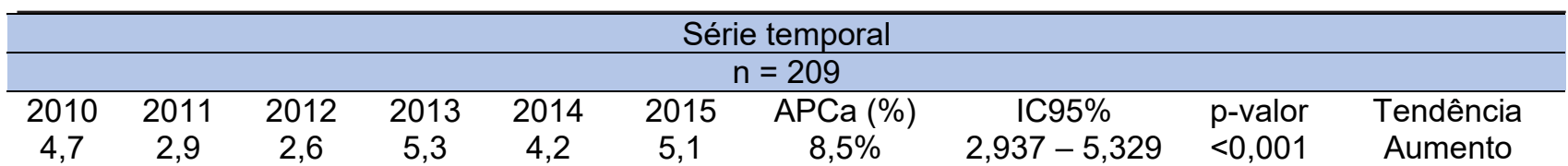

APCa: annual percent change ou variação percentual anual. IC: Intervalo de confiança.

Fonte: Registro Hospitalar de Câncer do Instituto Nacional do Câncer (RHC/INCA). 
de informação, foi observada uma taxa de incidência específica para o câncer de próstata, entre os homens de 20 a 59 anos, no período de 2010 a 2015 de, aproximadamente, 25 para cada 100.000 homens. A Tabela 2 apresenta a evolução temporal das taxas de incidência de câncer de próstata no Estado de Alagoas. Observou-se um crescimento de $8,5 \%$ na taxa de incidência de casos de câncer de próstata nos homens de 20 a 59 anos, entre 2010 a 2015, tendo em vista que passou de 4,7/100 mil habitantes em 2010 para 5,1/100 mil habitantes em 2015, com tendência crescente e significativa.

Os dados sobre mortalidade para câncer no Estado de Alagoas foram obtidos por meio do sistema de informação nacional. Entre as informações observadas, verificou-

Tabela 3. Características sociodemográficas dos óbitos por câncer de próstata em homens de 20 a 59 anos no período 2010 a 2015, Alagoas, Brasil

\begin{tabular}{|c|c|c|}
\hline Variáveis & $\mathrm{n}$ & $\mathrm{N}^{\circ}$ de óbitos com câncer de próstata (\%) \\
\hline TOTAL & 54 & 100,0 \\
\hline \multicolumn{3}{|l|}{ Faixa etária } \\
\hline$<40$ & - & - \\
\hline 40 a 44 & 02 & 3,7 \\
\hline 45 a 49 & 04 & 7,4 \\
\hline 50 a 54 & 17 & 31,5 \\
\hline 55 a 59 & 31 & 57,4 \\
\hline \multicolumn{3}{|l|}{ Raça/cor } \\
\hline Branca & 07 & 13,0 \\
\hline Parda & 35 & 64,8 \\
\hline Preta & 04 & 7,4 \\
\hline Sem Informação & 08 & 14,8 \\
\hline \multicolumn{3}{|l|}{ Escolaridade } \\
\hline Nenhuma & 08 & 14,8 \\
\hline Menos de 8 anos & 11 & 20,4 \\
\hline 8 anos ou mais & 09 & 16,7 \\
\hline Ignorado & 26 & 48,1 \\
\hline \multicolumn{3}{|l|}{ Estado civil } \\
\hline Casado & 24 & 44,4 \\
\hline Solteiro & 14 & 26,0 \\
\hline Viúvo & 02 & 3,7 \\
\hline Outro & 01 & 1,8 \\
\hline Sem Informação & 13 & 24,1 \\
\hline \multicolumn{3}{|c|}{ Região de saúde de residência } \\
\hline $1^{\text {a }}$ Região & 26 & 48,1 \\
\hline $2^{\mathrm{a}}$ Região & - & 0,0 \\
\hline $3^{\text {a Região }}$ & 02 & 3,7 \\
\hline $4^{a}$ Região & - & 0,0 \\
\hline $5^{\text {a Região }}$ & 03 & 5,5 \\
\hline $6^{\text {a Região }}$ & 06 & 11,1 \\
\hline $7^{\text {a }}$ Região & 09 & 17,0 \\
\hline $8^{\mathrm{a}}$ Região & 04 & 7,4 \\
\hline 9ª Região & 01 & 1,9 \\
\hline $10^{\text {a }}$ Região & 03 & 5,5 \\
\hline
\end{tabular}


se que a mortalidade por neoplasia maligna em Alagoas, no período de 2010 a 2015, apresentou uma ocorrência de 54 óbitos em decorrência do câncer de próstata em homens adultos.

$\mathrm{Na}$ Tabela 3 é possível identificar as características sociodemográficas dos óbitos por câncer de próstata em Alagoas entre os homens com idade entre 20 e 59 anos, no período estudado. Observou-se que entre os óbitos por câncer de próstata, as maiores proporções foram encontradas na faixa etária 55 a 59 anos $(57,4 \%)$, em indivíduos da raça/cor parda $(64,8 \%)$, que tiveram a escolaridade ignorada $(48,1 \%)$, casados $(44,4 \%)$ e residentes na $1^{\text {a }}$ Região de Saúde do Estado de Alagoas (48,1\%).

Diante dos registros no sistema de informação, foi observada uma taxa de mortalidade específica para câncer de próstata, entre os homens de 20 a 59 anos, no período de 2010 a 2015 de aproximadamente 6,5 para cada 100.000 homens. A Tabela 4 apresenta a evolução temporal das taxas de mortalidade de câncer de próstata no Estado de Alagoas. Observou-se um crescimento de $77,8 \%$ na taxa de mortalidade por câncer de próstata nos homens de 20 a 59 anos, entre
2010 a 2015 , tendo em vista que passou de 0,9/100 mil habitantes em 2010 para 1,6/100 mil habitantes em 2015, com tendência crescente e significativa.

\section{DISCUSSÃO}

O câncer de próstata é uma das neoplasias malignas que mais acometem homens em todas as regiões do Brasil e, em virtude de sua tendência de crescimento e representatividade nas condições de saúde da população masculina, tem impulsionado ações e políticas públicas, com foco na prevenção, na correção e de promoção da saúde desses indivíduos ${ }^{3}$.

Considerando a maior concentração dos casos de câncer de próstata em homens de idade mais avançada, grande parte dos estudos sobre o câncer de próstata concentram seus esforços nas estimativas e nos aspectos envolvidos para este seguimento populacional ${ }^{10-12}$. Poucos são os estudos que buscam identificar a tendência do câncer de próstata em homens mais jovens, tendo em vista a sua baixa incidência, no entanto, é preciso evidenciar que o câncer de próstata apresenta longo seguimento e,

Tabela 4. Tendência da taxa de mortalidade para câncer de próstata, em homens de 20 a 59 anos, no período de 2010 a 2015, Alagoas, Brasil

\begin{tabular}{cccccccccc}
\hline \multicolumn{10}{c}{ Série temporal } \\
\hline \multicolumn{10}{c}{$\mathrm{n}=54$} \\
0,9 & 2011 & 2012 & 2013 & 2014 & 2015 & APCa (\%) & IC95\% & p-valor & Tendência \\
& 1,1 & 1,3 & 0,6 & 0,9 & 1,6 & $77,8 \%$ & $0,699-1,434$ & 0,001 & Aumento
\end{tabular}

APCa: annual percent change ou variação percentual anual. IC: Intervalo de confiança.

Fonte: Sistema de Informação sobre Mortalidade do Sistema Único de Saúde (SIM/SUS). 
com isso, torna-se necessário o olhar atento também para a saúde do homem de faixa etária mais jovem.

Nesta perspectiva, este estudo buscou identificar a morbimortalidade por câncer de próstata, em homens de 20 a 59 anos, no Estado de Alagoas. Foi observado que no período estudado, a proporção de casos novos de câncer de próstata nesta faixa etária, em Alagoas, foi a menor quando comparada aos demais estados do Nordeste, considerando em números absolutos (Bahia=2.757; Pernambuco=850; Ceará $=789$; Rio Grande do Norte $=651$; Maranhão=387; Paraíba $=344 ; \quad$ Piauí=285; Sergipe $=216)^{4}$.

Levando em consideração que o câncer de próstata tem curso prolongado e que a presença de sinais e sintomas pode ocorrer a partir do comprometimento do órgão afetado, o menor número de casos de câncer de próstata na população estudada pode estar relacionado a uma subnotificação dos casos. Dessa forma, é importante que os profissionais de saúde estejam atentos durante os cuidados à saúde do homem, para o diagnóstico precoce da doença.

Ao identificar o perfil sociodemográfico dos casos novos de câncer próstata em Alagoas, este estudo observou a maior concentração de casos em homens mais velhos quando comparados aos mais novos. Evidências científicas ${ }^{3,6,7,13,14}$ discutem essa relação do $\mathrm{CaP}$ com idades mais avançadas e em virtude de sua pequena expressividade populacional, estimada em uma taxa de diagnóstico de menos de $1 \%$, desconsideram a relevância desse tipo de câncer em homens com menos de 60 anos.
No entanto, torna-se importante destacar que $10,0 \%$ dos casos observados nesta pesquisa foram em homens com menos de 50 anos, resultado corroborado por pesquisa desenvolvida a partir do pareamento dos sistemas de informação de saúde do país, buscando conhecer o perfil dos pacientes com câncer de próstata diagnosticados e tratados ambulatorialmente no SUS, que verificou a incidência de $10,7 \%$ deste tipo de câncer na faixa etária de 20 aos 59 anos no Brasil ${ }^{15}$.

O acompanhamento da evolução dos casos de câncer de próstata em homens mais jovens torna-se importante, principalmente, quando se considera os aspectos evolutivos da doença e os impactos econômicos para o sistema de saúde ${ }^{4}$, ressaltando a relevância do diagnóstico precoce como estratégia de obtenção de prognósticos satisfatórios e de redução das taxas de evolução para óbito por essa patologia.

Outra característica observada por este estudo foi a presença de maior proporção de casos de câncer de próstata entre homens da raça/cor parda. Alguns estudos têm relatado maior ocorrência de câncer de próstata na população negra, considerando pretos e pardos ${ }^{16-18}$. Pesquisa realizada no município de Ipirá (BA) verificou uma incidência de câncer de próstata nove vezes maior entre os negros quando comparados aos brancos ${ }^{17}$. Em estudos internacionais, menores taxas de incidência de câncer de próstata foram observadas em homens asiáticos, particularmente, na Índia, na China e no Japão e taxas mais elevadas foram verificadas em homens negros ${ }^{18}$.

Apesar da literatura pertinente 
apresentar que homens afro-americanos tenham entre 1,3 e 3,0 vezes mais risco de desenvolver o câncer de próstata quando comparados aos caucasianos e que homens negros (independentemente da raça negraafricana ou negra-caribenha) tenham 3,0 vezes mais risco de desenvolver câncer de próstata que homens brancos ${ }^{19}$, tornase difícil discutir aspectos relacionados à raça/cor nos padrões de morbimortalidade da população brasileira, em virtude da miscigenação da população como também da classificação se basear em autorrelato.

Outra característica identificada neste estudo foi quanto à escolaridade dos homens que tiveram diagnóstico de câncer de próstata no Estado de Alagoas, no período de 2010 a 2015 . Observou-se que a maioria dos homens diagnosticados apresentavam menos de nove anos de estudo, resultado que corrobora com outras pesquisas sobre escolaridade em indivíduos com câncer de próstata $^{12,20}$. Estudo populacional realizado em São Paulo $^{12}$ verificou a existência de associação estatisticamente significativa entre a não realização de exames preventivos para o câncer de próstata em homens com menos de nove anos de estudo. Esse resultado pode influenciar a busca pelo serviço de saúde para o acompanhamento preventivo, como também pode interferir de forma significativa no diagnóstico precoce da doença e no início de seu tratamento. Por isso, o estímulo à participação da população masculina nos serviços de saúde torna-se importante para o rastreamento e cuidado efetivo a saúde desse grupo populacional.

Neste estudo também foi observado que, entre as informações registradas de casos novos de câncer de próstata, as maiores proporções ocorreram em homens casados, resultado que corrobora com achados de outras pesquisas da literatura ${ }^{17,21-23}$. Pesquisa realizada em Vitória, no Estado do Espírito Santo, com indivíduos que receberam atendimento na rede estadual de oncologia, observou que $77,0 \%$ dos homens com diagnóstico de câncer de próstata atendidos no serviço eram casados. Sabe-se que culturalmente o homem tende a não frequentar de forma preventiva os serviços de saúde, no entanto, aqueles que apresentam cônjuge podem ser estimulados ou mesmo levados ao atendimento de saúde e, com isso, apresentar mais probabilidade de se submeter a um tratamento ${ }^{23}$.

Embora a etiologia do câncer de próstata ainda permaneça pouco compreendida, estudos sobre os fatores de risco são importantes para o planejamento em saúde e o estímulo a comportamentos e hábitos saudáveis. Nesta perspectiva, foi observado nesta pesquisa que a maioria dos homens diagnosticados com câncer de próstata no Estado de Alagoas consumia ou já consumiram bebida alcoólica. Esse resultado corrobora com estudo realizado em Londrina ${ }^{10}$, com homens diagnosticados com câncer de próstata acompanhados em Hospital Universitário, que identificou o uso declarado de bebida alcoólica em $67,0 \%$ dos entrevistados. A identificação dos fatores de risco presentes na população masculina é condição importante para o planejamento do cuidado em saúde e a organização de ações que busquem minimizar os efeitos negativos de hábitos, muitas vezes, existentes por influência cultural. 
Outro fator de risco importante a ser considerado e que apresenta efeitos negativos sobre a saúde da população é o uso de tabaco, tendo em vista a presença de aminas aromáticas que podem facilitar o desenvolvimento do câncer de próstata ${ }^{10}$. Vários estudos têm verificado a relação entre o tabagismo e o câncer ${ }^{10,12,24}$ e quando o uso está associado a longo período de tempo, os riscos são ainda maiores. Pesquisa realizada com homens diagnosticados com câncer de próstata, acompanhados em Hospital Universitário, identificou que $28,0 \%$ dos entrevistados relataram uso de tabaco por mais de 51 anos e esse período prolongado pode ter potencializado o risco para 0 desenvolvimento de câncer de próstata em $46,0 \%$ dos sujeitos que fumaram ou ainda fumam $^{10}$. O enfrentamento dos fatores de risco à saúde da população deve ser uma estratégia das políticas públicas de saúde, tendo em vista o seu potencial cancerígeno.

Apesar das informações obtidas sobre o uso de bebida alcoólica e de tabaco neste estudo, merece destaque o número elevado de ausência de informação sobre esses fatores de risco. Torna-se importante reforçar o necessário preenchimento das informações que circundam o câncer de próstata, tendo em vista seu potencial influenciador da construção do raciocínio no diagnóstico do câncer de próstata, bem como para esclarecer aspectos etiológicos e epidemiológicos dos acometidos, de modo que a presença dessas incompletudes pode interferir diretamente para análise dos fatores de risco relacionados à doença.

Além dos fatores de risco que devem ser levantados para a compreensão da etiologia das doenças, a identificação da presença de marcadores de risco também é relevante para a análise dos aspectos envolvidos no adoecimento por câncer de próstata. Neste estudo foi possível observar a maior proporção de pacientes sem histórico familiar da doença, resultado que difere de achados presentes na literatura ${ }^{10,16,25}$.

Artigo de revisão sobre câncer de próstata evidenciou que com a existência de um parente de primeiro grau com a doença, tem-se um risco, no mínimo, duas vezes maior de desenvolvimento de câncer de próstata e se dois ou mais familiares apresentarem este histórico, esse risco aumenta de cinco a onze vezes ${ }^{16}$. Torna-se conveniente destacar que, no presente estudo, $32,0 \%$ dos casos estudados não apresentavam informações sobre o histórico familiar da doença, o que pode ter influenciado uma subestimação do número de indivíduos com câncer de próstata com histórico familiar, condição que deve ser abordada durante o cuidado a saúde do homem, considerando o risco associado ao desenvolvimento de câncer de próstata dependente do grau de parentesco e do número de parentes afetados.

A análise de tendência temporal da morbidade para câncer de próstata entre homens de 20 a 59 anos no Estado de Alagoas, no período de 2010 a 2015, tem revelado que, apesar de ainda apresentar pequena incidência, o número de casos novos sofreu uma variação percentual anual crescente, o que traduz a necessidade de vigilância da doença entre os homens neste seguimento populacional. 
O presente estudo buscou traçar um perfil epidemiológico da morbimortalidade para o câncer de próstata entre homens de 20 a 59 anos no Estado de Alagoas, no entanto, em virtude de sua abordagem, apresenta limitações metodológicas, a saber: i) o uso de dados secundários que não permite ao pesquisador controle de possíveis erros decorrentes de digitação, registro, omissão no preenchimento dos campos e possíveis subnotificações; ii) importante número de variáveis com preenchimento ignorado e/ ou sem informação, decorrente da falta de padronização na coleta e sistematização dos dados pelos serviços; iii) carência de estudos epidemiológicos que abordem a população de interesse (homens de 20 a 59 anos) permitindo uma discussão mais aprofundada dos dados com outras localidades do país; e iv) possibilidade de confundimento residual devido à ausência de controle das associações por variáveis não disponíveis nas bases de dados e/ou pela própria dificuldade em atribuir ao nível individual às mesmas associações ecológicas para o espaço-tempo aqui analisado. Porém, mesmo com essas considerações, é importante salientar que o estudo tomou como referência dados provenientes de fontes nacionais de informação, acreditadas e mantidas pelo Ministério da Saúde e que são de preenchimento obrigatório em todos os serviços que recebem recursos do SUS para o tratamento de pacientes oncológicos. Não menos importante, cabe mencionar que os estudos ecológicos são uma alternativa de baixo custo e de rápida execução para estabelecer as correlações de interesse para a saúde pública.

\section{CONCLUSÃO}

Este estudo observou que o perfil de morbimortalidade relacionado ao câncer de próstata em homens de 20 a 59 anos, no período de 2010 a 2015, apresentou maiores proporções de acometimento em indivíduos acima de 50 anos, pardos, com baixa escolaridade, casados, com histórico de consumo de bebida e tabaco em algum momento, sem histórico familiar da doença e que, espacialmente, estavam concentrados nas maiores cidades do Estado de Alagoas. A tendência de óbitos e de diagnósticos para o recorte etário adotado foi crescente, com variações percentuais anuais estatisticamente relevantes e valores indicando inclinação temporal de aumento.

Novos trabalhos precisam ser estabelecidos, com fins de avaliar a possível relevância epidemiológica do câncer de próstata em homens adultos em outros cenários do país, como é o caso de outros estados e regiões. Estudos dessa natureza podem contribuir para o conhecimento do comportamento dessa e de outras neoplasias em um espaço-tempo e possibilitar, assim, a tomada de decisão de administradores públicos quanto à necessidade de adoção de medidas de intervenção no âmbito da saúde do homem ou mesmo de (re)planejamento da rede de cuidados com fins de melhor atender esse segmento populacional. 


\section{REFERÊNCIAS}

1. World Health Organization. World Cancer Report 2014. Lyon: World Health Organization; 2014.

2. Instituto Nacional de Câncer (INCA). CÂNCER: Magnitude do problema [internet]. Rio de Janeiro; [Acesso em: 16 dez. 2019].

3. Ministério da Saúde (BR). Secretaria de Atenção à Saúde. Política Nacional de Atenção Integral à Saúde do Homem: princípios e diretrizes. Brasília: Ministério da Saúde, 2009. p. 7-9.

4. Instituto Nacional de Câncer (INCA). Estimativa 2020: incidência de câncer no Brasil. [internet]. Rio de Janeiro; [Acesso em: 20 dez. 2019].

5. Ministério da Saúde (BR). DATASUS. Informações de Saúde: Mortalidade 2010-2015. [internet]. Rio de Janeiro; [Acesso em: 20 dez. 2019].

6. Medeiros AP, Menezes MFB, Napoleão AA. Fatores de risco e medidas de prevenção do câncer de próstata: subsídios para a enfermagem. Rev. bras. enferm. 2011; 64(2):385-388.

7. Sociedade Brasileira de Urologia. Nota Oficial: Rastreamento do Câncer de Próstata. [internet]. 2018. [Acesso em: 25 dez. 2019].

8. Guerra MR, Gallo CVM, Mendonça GAS. Risco de câncer no Brasil: tendências e estudos epidemiológicos mais recentes. Rev Bras Cancerologia 2005; 51(3):227-234.

9. Antunes YPPV, Bugnao DDG, Gilio A, Kaliks RA, Karnakis T, Pontes LB. Características clínicas e de sobrevida global em pacientes oncológicos idosos num centro oncológico terciário. Einstein. 2015;13(4):Epud,11.

10. Fernandes MV, Martins JT, Cardelli AAM, Marcon SS, Ribeiro RP. Perfil epidemiológico do homem com câncer de próstata atendido em um Hospital Universitário. Cogitare Enferm. 2014;19(2):333-340.

11. Souza KS, Miranda FSL, Silva MRF, Costa GS, Pereira RB et al. Tendência de mortalidade por câncer de próstata na região Nordeste do Brasil, 1996-2014. Rev. Rios Saúde. 2018; 1(2):18-28.

12. Amorim VMSL, Barros MBA, César CLG, Goldbaum M, Carandina L, Alves MCGP. Fatores associados à realização dos exames de rastreamento para o câncer de próstata: um estudo de base populacional. Cad. Saúde Pública. 2011; 27(2):347-356.
13. Ministério da Saúde (BR). INCA. Registros Hospitalares de Câncer (RHC). [internet]. Rio de Janeiro; [Acesso em: 18 dez. 2017].

14. Braga SFM. Câncer da próstata: sobrevida, fatores associados ao risco de óbito e tendência de mortalidade no Brasil. Belo Horizonte. Tese [Doutorado em Saúde Pública]. Universidade Federal de Minas Gerais; 2003.

15. Braga SFM, Souza MC, Oliveira RR, Andrade EIG, Acurcio FA, Cherchiglia ML. Patient survival and risk of death after prostate cancer treatment in the Brazilian Unifi ed Health System. Rev de Saude Publica. 2017;51(0):46.

16. Johns LE, Houlston RS. A systematic review and metaanalysis of familial prostate cancer risk. BJU Int. 2003 91(9):789-794.

17. Gonçalves IR, Padovani C, Popim RC. Caracterização epidemiológica e demográfica de homens com câncer de próstata. Ciênc. saúde coletiva. 2008;13(4):1337-1342.

18. Metcalfe C, Patel B, Evans S, Ibrahim F, Anson $\mathrm{K}$, Chinegwundoh $\mathrm{F}$ et al. The risk of prostate cancer amongst South Asian men in southern England: the PROCESS cohort study. BJU Int. 2008;102(10):14071412.

19. Paschoalin EL, Martins AC, Pastorello $M$, Sândis KA, Maciel LM, Silva WA Jr, Zago Ma, Bessa J Jr. Send to Racial influence on the prevalence of prostate carcinoma in Brazilian volunteers. Int Braz J Urol. 2003; 29(4):300-305.

20. Migowski A, Silva GA. Sobrevida e fatores prognósticos de pacientes com câncer de próstata clinicamente localizado. Rev Saúde Pública. 2010; 44(2):344-352.

21. Zacchi SR, Amorim MHC, Souza MAC, Miotto MHMB, Zandonade E. Associação de variáveis sociodemográficas e clínicas com o estadiamento inicial em homens com câncer de próstata. Cad Saúde Colet. 2014; 22(1):93-100

22. Lima AP, Lini EV, Giacomazzi RB, Dellani MP, Portella MR, Doring M. Prevalence and factors associated with the performance of prostate cancer screening in the elderly: a population-based study. Rev Bra. Geriatr Gerontol. 2018; 21(1):53-59.

23. Modena CM, Martins AM, Ribeiro RBN, Almeida SSL. Os homens e o adoecimento por câncer: um olhar sobre a produção científica brasileira. Rev Baiana de Saúde Pública. 2013; 37(3):644-660. 
24. Czorny RCN, Pinto MH, Pompeo DA, Bereta D, Cardoso LV, Silva DM. Fatores de risco para o câncer de próstata: população de uma unidade básica de saúde*. Cogitare Enferm.;2017; 22(4): e51823.

25. Rhoden EL, Averbeck MA. Câncer de próstata localizado. Rev da AMRIGS. 2010; 54(1):92-99.

\section{CORRESPONDÊNCIA}

Claudio José dos Santos Júnior. Universidade Estadual de Ciências da Saúde de Alagoas (UNCISAL) E-mail: claudiosantos_al@hotmail.com. 